\title{
Autopercepção de estresse em idosos submetidos a tratamento hemodialítico em um centro de diálise
}

RESUMO | O estresse em portadores de doença renal crônica é parte de um ciclo inerente a esta doença. Objetivo: verificar a autopercepção de estresse em idosos submetidos ao tratamento hemodialítico. Métodos: foi realizado um estudo transversal, mediante entrevista semiestruturada com idosos em um centro de diálise. O estudo contou com 18 idosos, dos quais $55 \%$ são do sexo masculino. A média da idade encontrada foi 69,8 anos ( $\pm 8,11$ anos), com predomínio da faixa etária de 60 a 69 anos. Conclusão: Este estudo pode verificar que o sexo e a faixa etária não mostraram associação significativa com a presença de estresse autopercebido entre idosos com doença renal crônica que realizam tratamento hemodialítico. Ainda que o estresse seja uma condição comum nos idosos, seu significado é bastante variável naqueles que realizam tratamento hemodialítico, tendo em vista a multiplicidade de fatores que podem interagir entre si na causalidade dessa condição.

Palavras-chaves: Insuficiência renal crônica, Estresse, Hemodiálise.

ABSTRACT I Stress in patients with chronic kidney disease is part of a cycle inherent in this disease. The objective of this study is to verify the self-perception of stress in the elderly undergoing hemodialysis treatment. A cross-sectional study was conducted through a semi-structured interview with the elderly in a dialysis center. The study had 18 elderly people, 55\% of whom were male. The mean age found was 69.8 years ( \pm 8.11 years), with a predominance of the age group from 60 to 69 years. This study can verify that gender and age group did not show a significant association with the presence of self-perceived stress among elderly patients with chronic renal disease who undergo hemodialysis treatment. Although stress is a common condition in the elderly, its meaning is quite variable in those who undergo hemodialysis treatment, considering the multiplicity of factors that may interact with each other in the causality of this condition.

Keywords: Chronic renal failure, Stress, Hemodialysis.

RESUMEN | El estrés en pacientes con enfermedad renal crónica es parte de un ciclo inherente a esta enfermedad. Objetivo: verificar la autopercepción de estrés en ancianos en tratamiento de hemodiálisis. Métodos: se realizó un estudio transversal a través de entrevistas semiestructuradas con ancianos en un centro de diálisis. El estudio incluyó a 18 ancianos, de los cuales el 55\% son hombres. La edad promedio encontrada fue de 69.8 años ( \pm 8.11 años), con un predominio del grupo de edad de 60 a 69 años. Conclusión: Este estudio puede verificar que el género y el grupo de edad no mostraron una asociación significativa con la presencia de estrés autopercibido entre los ancianos con enfermedad renal crónica sometidos a tratamiento de hemodiálisis. Aunque el estrés es una condición común en los ancianos, su significado es bastante variable en aquellos que reciben tratamiento de hemodiálisis, considerando la multiplicidad de factores que pueden interactuar entre sí en la causalidad de esta condición.

Palabras claves: Insuficiencia renal crónica, Estrés, Hemodiálisis.

\section{Clarice Santana Milagres}

Enfermeira. Doutora em Saúde Coletiva pela Universidade Estadual de Campinas UNICAMP. Docente do Centro Universitário Hermínio Ometto - UNIARARAS.

\section{Jucilene Casati Lodi}

Enfermeira. Doutoranda em Saúde Coletiva pela Faculdade de Odontologia de Piracicaba FOP/UNICAMP.

Recebido em: 28/07/2019

Aprovado em: 18/09/2019

INTRODUÇÃO

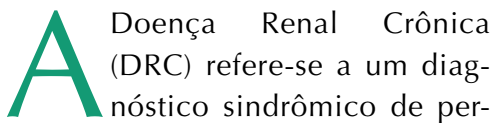

da progressiva e irreversível da função renal de depuração, ou seja, da filtração glomerular, e está associada à alta morbidade e mortalidade, com aumento progressivo nas populações mundiais ${ }^{1}$.

A Sociedade Brasileira de Nefrologia (SBN) estima que 126.583 mil pacientes estão em tratamento dialítico, com uma taxa de ocupação de $85 \%$ dos centros de diálise em funcionamento atualmente. A taxa de prevalência do tratamento dialítico em 2017, foi de 671 pacientes por um milhão da população no Estado de São Paulo, sendo este valor o mais significativo da região Sudeste. Estima-se que, 40.307 pacientes iniciaram o tratamento no ano referido. Quanto ao sexo, $58 \%$ são do sexo masculino e $42 \%$ do feminino; e a faixa etária com maior concentração de pacientes compreende idades de 20 a 64 anos $^{2}$.

O tratamento depende da evolução da doença, o aumento da sintomatologia e comprometimento das condições de saúde e agravos das doenças crônicas, que contribuem para o início da Terapia Renal Substitutiva (TRS). As modalidades existentes são: hemodiálise; diálise peritoneal e transplante renal ${ }^{3}$. Segundo o Censo Brasileiro de Diálise, no ano de 2017, 93,1\% dos pacientes realizavam tratamento por hemodiálise e $6,9 \%$ por diálise peritoneal ${ }^{2}$. 
A TRS da modalidade hemodiálise acontece mediante a circulação extracorpórea, realizada entre membranas derivadas de celulose que atuam como membrana semipermeável. É um procedimento que remove os produtos urêmicos acumulados, o excesso de água e restabelece o equilíbrio acidobásico e eletrolítico do organismo ${ }^{1,3}$.

$\mathrm{Na}$ literatura encontram-se estudos que avaliam as limitações (físicas, restrições laborais e perdas sociais) envolvidas no cotidiano dos pacientes renais crônicos e que buscam compreender fatores relacionados à qualidade de vida (estágio da doença, componente físico e mental; hospitalização e tempo de diálise), às comorbidade mentais (estresse) que influenciam a saúde e o cotidiano desses pacientes ${ }^{4-6}$.

Em relação ao estresse, verifica-se que este é comum em pacientes portadores de Insuficiência Renal Crônica (IRC) e que realizam tratamento hemodialítico. Fatores associados às diversas restrições advindas do tratamento, exemplificadas pela dificuldade deste paciente viajar para outro estado ou município, dependentes da concessão prévia de vaga para dialisar em outro centro de diálise; alteração na alimentação com restrições ao sódio, fósforo, potássio; e limitação na ingesta hídrica de acordo com a diurese residual apresentada ou não por ser portador de insuficiência renal ${ }^{7,8}$. Portanto, o estresse em pacientes idosos que possuem doença crônica se torna cada vez mais frequente. Esta causa também está ligada à classificação da doença que vai de uma escala de I ao V, onde a cada nível é estimado um tratamento mais rigoroso, podendo levar o portador de doença renal às diversas fases da doença crônica, em especial a fase denominada de negação e/ou fase da revolta, e que influencia cada vez mais o nível de estresse na faixa etária acima dos 60 anos e mais ${ }^{9}$.

Ao considerar que o estresse em portadores de doença renal crônica é parte de um ciclo inerente à doença, sugere como pergunta norteadora da pesquisa: o idoso tem percepção que o tratamento hemodialítico possa provocar estresse? Dessa forma, o estudo teve como objetivo verificar a autopercepção de estresse em idosos submetidos ao tratamento hemodialítico em um centro de diálise. Segundo a literatura, há reduzido número de estudo que analisa o estresse a partir da autopercepção do idoso, em especial, o idoso portador de doença renal crônica em tratamento hemodialítico.

\section{MÉTODOS}

Pesquisa descritiva exploratória, com abordagem quantitativa, utilizadas entrevistas semiestruturadas com 18 pacientes idosos (a partir de 60 anos), que aceitaram participar da pesquisa e que foram acometidos por IRC e submetidos ao tratamento hemodialítico em um centro privado de diálise no município de Rio Claro/SP.

A coleta de dados ocorreu em um único dia de fevereiro de 2018 (devido a liberação da instituição) em pacientes que realizavam hemodiálise. O critério de inclusão: pacientes idosos acima de 60 anos, que no dia da entrevista da pesquisadora, estivessem presentes na clínica e aceitassem participar. Critérios de exclusão: pacientes com menos de 60 anos e que não estivessem na clínica no dia da entrevista.

A partir da assinatura do Termo de Consentimento Livre e Esclarecido (TCLE), foi aplicado os questionários de identificação e questões semiestruturadas relativas ao tratamento hemodialítico.

Seguiam-se os questionários de identificação (sexo e idade) e a utilização do questionário extraído da Escala de Estresse Percebido, validado por Luft (2007), no qual contem 14 questões, onde são questionados aos idosos seus pensamentos e sentimentos. Em seguida foi utilizada uma escala do tipo $\mathrm{Li}^{-}$ kert que varia de 0 a 4 pontos, sendo 0 : nunca, 1: quase nunca, 2: as vezes, 3 : quase sempre e 4: sempre (10).

A Escala de Estresse Percebido utilizou os seguintes questionamentos: estar triste por causa de algo que aconteceu inesperadamente, sentir-se incapaz de controlar as coisas importantes da vida, sentir-se nervoso e "estressa-

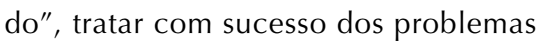
difíceis, lidar bem as mudanças importantes que estão ocorrendo, sentir-se confiante na habilidade de resolver problemas pessoais, sentir que as coisas estão acontecendo de acordo com a sua vontade, achar que não conseguiria lidar com todas as coisas tem que fazer, conseguir controlar as irritações, sentir que as coisas estão sob o seu controle, ficar irritado porque as coisas que acontecem estão fora do seu controle, encontrar-se pensando sobre as coisas que deve fazer, conseguir controlar a maneira como gasta seu tempo e sentir que as dificuldades se acumulam a ponto de acreditar que não pode superá-las ${ }^{10}$.

Foi utilizado os softwares STATA 9.1. A distribuição e frequência das variáveis do estudo foi verificada. As análises bivariadas incluíram o teste do qui-quadrado para variáveis categóricas, no nível de significância de $p<0,05$.

Para a análise da Escala de Estresse Percebido (PSS), que pode variar de 0 a 56 pontos, foi calculado ou considerado a média (28 pontos) da seguinte forma: menor que 28 pontos é considerado baixo estresse, e igual ou superior a 28 pontos alto estresse.

Esta pesquisa foi aprovada pelo Comitê de Ética em Pesquisa do Centro Universitário Hermínio Ometto - UNIARARAS, conforme número do CAAE - 73817917.0.0000.5385.

\section{RESULTADOS}

Participaram da pesquisa 18 idosos, sendo, 55\% do gênero masculino. A mé- 
dia da idade encontrada foi 69,8 anos (DP $= \pm 8,11$ anos), no qual houve predomínio da faixa etária de 60 a 69 anos, correspondendo a 11 idosos dessa amostra.

Verificou-se dentre as atividades propostas pela PPS, somente nas atividades de dificuldades que se acumulam a ponto de acreditar que não pode superá-las, apresentaram maior frequência de idosos que relatam nunca ou quase nunca terem passado por essa sensação. Entre idosos que relataram maiores frequências de atividades propostas pelo instrumento, estão: tratar com sucesso dos problemas difíceis da vida, sentir que está lidando bem as mudanças importantes que estão ocorrendo, achar que não conseguiria lidar com todas as coisas que tem que realizar, con- seguir controlar as irritações em sua vida e ter se encontrado pensando sobre as coisas que deve fazer. Todos os participantes, em diferentes quantidades, trataram com sucesso dos problemas difíceis de sua vida, em especial, com maiores frequências para respostas como sempre ou quase sempre (tabela 1).

Foi observado que $55 \%$ dos entrevistados referem que sempre ou quase sempre se sentem tristes por algo que aconteceu inesperadamente e sente que as coisas estão sobre seu controle; $77 \%$ tem sentido que estão lidando bem com as mudanças que estão ocorrendo e se encontram pensando sobre as coisas que tem que fazer; sobre a habilidade em resolver problemas pessoais, aproximadamente $67 \%$ respon- deram que sempre ou quase sempre conseguem realiza-lo, enquanto $72 \%$ dos idosos relataram que sempre ou quase sempre sentiam que não conseguiriam lidar com todos as coisas que tem que fazer ou conseguiriam controlar suas irritações. Por fim, quando questionados sobre a maneira como gastam seu tempo, $61 \%$ da amostra relataram que consegue controlar o gasto do seu tempo.

Conforme a pesquisa nenhum dos pacientes tem conseguido tratar com sucesso os problemas difíceis da vida; $50 \%$ dos entrevistados tem se sentido incapaz de controlar as coisas importantes em sua vida e aproximadamente $67 \%$ tem sentido que as dificuldades estão se acumulando a ponto de acreditar que não podia superar.

\section{Tabela 1. Autoaplicação da Escala de Estresse Percebido em idosos, 2018.}

\begin{tabular}{|c|c|c|c|}
\hline Atividades & $\begin{array}{l}\text { Nunca/ Quase nunca } \\
\qquad \mathrm{N}(\%)\end{array}$ & $\begin{array}{l}\text { Às vezes } \\
\mathrm{N}(\%)\end{array}$ & $\begin{array}{l}\text { Sempre Quase/ } \\
\text { Sempre } \\
\text { N (\%) }\end{array}$ \\
\hline $\begin{array}{l}\text { Você tem ficado triste por causa de algo que aconteceu } \\
\text { inesperadamente? }\end{array}$ & $6(33,4 \%)$ & $2(11,11 \%)$ & $10(55,6 \%)$ \\
\hline $\begin{array}{l}\text { Você tem se sentido incapaz de controlar as coisas impor- } \\
\text { tantes em sua vida? }\end{array}$ & $9(50 \%)$ & $1(5,5 \%)$ & $8(44,5 \%)$ \\
\hline Você tem se sentido nervoso e "estressado"? & $5(27,8 \%)$ & $6(33,4 \%)$ & $7(38,8 \%)$ \\
\hline $\begin{array}{l}\text { Você tem tratado com sucesso dos problemas difíceis da } \\
\text { vida? }\end{array}$ & 0 & $2(11,1 \%)$ & $17(88,9 \%)$ \\
\hline $\begin{array}{l}\text { Você tem sentido que está lidando bem as mudanças } \\
\text { importantes que estão ocorrendo em sua vida? }\end{array}$ & $1(5,5 \%)$ & $3(16,7 \%)$ & $14(77,8 \%)$ \\
\hline $\begin{array}{l}\text { Você tem se sentido confiante na sua habilidade de resol- } \\
\text { ver problemas pessoais? }\end{array}$ & $5(27,8 \%)$ & $1(5,6 \%)$ & $12(66,7 \%)$ \\
\hline $\begin{array}{l}\text { Você tem sentido que as coisas estão acontecendo de } \\
\text { acordo com a sua vontade? }\end{array}$ & $6(33,4 \%)$ & $4(22,2 \%)$ & $8(44,5 \%)$ \\
\hline $\begin{array}{l}\text { Você tem achado que não conseguiria lidar com todas as } \\
\text { coisas que você tem que fazer? }\end{array}$ & $4(22,3 \%)$ & $1(5,6 \%)$ & $13(72,2 \%)$ \\
\hline Você tem conseguido controlar as irritações em sua vida? & $2(11,2 \%)$ & $3(16,7 \%)$ & $13(72,2 \%)$ \\
\hline Você tem sentido que as coisas estão sob o seu controle? & $5(27,8 \%)$ & $3(16,7 \%)$ & $10(55,5 \%)$ \\
\hline $\begin{array}{l}\text { Você tem ficado irritado porque as coisas que acontecem } \\
\text { estão fora do seu controle? }\end{array}$ & $6(33,4 \%)$ & $5(27,8 \%)$ & $7(39,00 \%)$ \\
\hline $\begin{array}{l}\text { Você tem se encontrado pensando sobre as coisas que } \\
\text { deve fazer? }\end{array}$ & $3(16,7 \%)$ & $1(5,50 \%)$ & $14(77,8 \%)$ \\
\hline $\begin{array}{l}\text { Você tem conseguido controlar a maneira como gasta seu } \\
\text { tempo? }\end{array}$ & $4(17,2 \%)$ & $3(16,7 \%)$ & $11(61,1 \%)$ \\
\hline $\begin{array}{l}\text { Você tem sentido que as dificuldades se acumulam a ponto } \\
\text { de você acreditar que não pode supera-las? }\end{array}$ & $12(66,8 \%)$ & $3(16,6 \%)$ & $3(16,6 \%)$ \\
\hline
\end{tabular}


A análise da ocorrência de estresse de acordo com o sexo revelou que $80 \%$ dos indivíduos que apresentavam alto estresse eram homens, enquanto entre as mulheres, esta frequência foi de $87,5 \%$. Em relação à idade, a faixa etária entre os idosos mais jovens, mostrou maior frequência de alto estresse. $\mathrm{O}$ teste qui-qua- drado não mostrou associação significativa entre a presença de estresse e o sexo, assim como em relação à variável idade (Tabela 2).

Tabela 2. Presença de estresse associado com a variável gênero e idade de idosos, Rio Claro, SP, 2018.

\begin{tabular}{|c|c|c|c|c|}
\hline \multirow{2}{*}{ Variáveis } & \multirow{2}{*}{$\begin{array}{c}\text { Amostra } \\
(n=18)\end{array}$} & \multicolumn{2}{|c|}{ Presença de Estresse } & \multirow{2}{*}{ p- valor } \\
\hline & & Alto & Baixo & \\
\hline \multicolumn{5}{|l|}{ Sexo } \\
\hline Feminino & $08(44,4 \%)$ & $07(87,5 \%)$ & $01(12,5 \%)$ & 0,83 \\
\hline \multicolumn{5}{|l|}{ Faixa etária } \\
\hline 71 a 80 & $04(22,2 \%)$ & $03(75 \%)$ & $01(25 \%)$ & 0,55 \\
\hline$\geq 81$ anos & $03(16,7 \%)$ & $02(66,7 \%)$ & $01(33,3 \%)$ & 0,89 \\
\hline
\end{tabular}

Fonte: Dados da pesquisa, 2018.

\section{DISCUSSÃO}

As doenças degenerativas como a Doença Renal Crônica são atualmente consideradas um dos grandes problemas de saúde pública, em especial, entre os idosos. Esta última é representada por um alto índice de morbimortalidade no Brasil e no mundo ${ }^{11}$. Segundo Draczevski e Teixeira (2011), a Doença Renal Crônica exige um tratamento de alto custo e complexidade e nos últimos anos, obteve um aumento significativo e preocupante para o Sistema Único de Saúde, decorrente da transição epidemiológica de doenças transmissíveis para doenças não transmissíveis, as quais contribuíram para o perfil de morbimortalidade ${ }^{12-14}$. Os idosos, sendo reconhecidos como grupo de risco para o desenvolvimento de doenças crônicas, conforme o perfil populacional e epidemiológico do país, colaboram no aumento dos fatores diversos desencadeantes para a progressão de uma diminuição da qualidade de vida, assim como aumento dos gastos financeiros associados à DRC ${ }^{11}$.

O estresse, definido como um conjunto de reações específicas e gerais do organismo frente à persistente estimulação da natureza aversiva é capaz de alterar o equilíbrio homeostático do corpo. No pa- ciente idoso e portador de doença renal crônica, submetido ao tratamento dialítico, as mudanças inerentes ao estresse podem ser acompanhadas por alterações bioquímicas, psicológicas, cognitivas e comportamentais, uma vez que, a dependência de uma máquina de diálise e da equipe que a manipula pode desencadear, por si só, um evento estressante ${ }^{15}$. Os idosos experimentam mudanças significativas, não apenas por possuírem a doença renal, mas também por possuírem maior risco de desenvolver perda de capacidade física, limitações na coordenação motora, dificuldades de locomoção e tomadas de decisão, necessitando de atenção especial ${ }^{9}$.

Pacientes acometidos por insuficiência renal estão mais suscetíveis ao alto nível de estresse, muitas vezes pela própria mudança no estilo de vida, no presente estudo, foram observadas elevadas frequências de idosos e idosas que apresentavam alto índice de estresse. Ao analisar o estresse relacionado ao sexo, Arreguy-Sena (2018), o estresse mais predominante no sexo masculino pode ser advindo de uma questão cultural ou até mesmo psicológica ${ }^{9}$. O presente estudo, mostrou que os idosos com doença renal crônica, submetidos ao tratamento hemodialítico, não apresentaram alto nível de estresse, assim como o trabalho proposto por Patrício et al (2016), que ao analisar idosos assistidos em unidades básicas de saúde na Paraíba, através da escala de estresse autopercebido não apresentaram alto nível de estresse, mesmo possuindo doenças crônicas incapacitantes e que levam à relativa dependência, como a doença rena crônica ${ }^{16}$.

A parceria da pessoa com a máquina produz uma ambiguidade de sensações, que, segundo Campos et al (2015) pode incorrer na dualidade raiva e gratidão, pois significa dependência e manutenção da vida. A representação de perda de liberdade atrelada à máquina de hemodiálise remete ao processo de estresse vivenciado rotineiramente por estes idosos, que ao internalizar o tratamento hemodialítico necessários à continuidade de vida e a progressão dos anos já vividos, trazem consigo a impossibilidade de desvincular-se da percepção de uma abreviação da vida ${ }^{17}$.

Segundo Mattos e Maruyama (2010), uma das maiores causas para a ocorrência do estresse em pacientes que realizam hemodiálise são as condições particulares no qual estão submetidos, como necessitar de acessar os serviços de saúde e o centro de diálise, controlar de forma rigorosa a dieta e a ingestão de líquidos, possuir restrição na execução de atividades laborais e par- 
ticipar de forma reduzida do orçamento doméstico. Este quadro configura como perdas que afetam pacientes e familiares no contexto da hemodiálise. Ao se tratar deste paciente, que, além de portador de doença renal crônica, também possui idade igual ou superior a 60 anos, o adoecimento e a necessidade de hemodiálise, repercute em diversas dimensões, como a pessoal, familiar e social ${ }^{18-20}$.

Em idosos, além das situações especiais vivenciadas pelos portadores de doença renal crônica, ainda há a falta de confiança em si mesmo para realizar ou resolver pendencias pessoais, como por exemplo, realizar as atividades básicas e avançadas de vida diária, como ir ao banheiro sozinho e preparar a própria comida, fazer compras, pagar contas e outras. Diante de atividades de vida diária e a impossibilidade de realização das mesmas de forma independente, os idosos tendem a apresentar-se nervosos. Por mais que nos centros de dialise se- jam oferecidos tratamentos psicológicos, a oferta de um acompanhamento no qual o estresse é trabalhado em associação com a progressão da idade avançada e ao tratamento, se mostra fundamental ao tentar redução no nível de estresse autopercebido, assim como poder melhorar o impacto que a doença e suas limitações podem desencadear na pessoa idosa ${ }^{20-21}$.

O presente estudo apresenta limitações, como o delineamento transversal, que não permite clarificar as relações temporais de causa e efeito entre o estresse autopercebido e demais fatores que podem desencadeá-lo diante de um tratamento de alta complexidade como a hemodiálise. Outra limitação do estudo foi a impossibilidade de caracterizar os dados socioeconômicos dos idosos selecionados no estudo. A despeito dessas limitações, foi possível identificar associações relevantes entre o estresse autopercebido e fatores envolvidos no mecanismo na superação do tratamento da doença renal crônica em idosos.

\section{CONCLUSÃO}

Este estudo apresentou poucos participantes, entretanto, pode verificar que o sexo e a faixa etária não mostraram associação significativa com a presença de estresse autopercebido entre idosos em tratamento de hemodiálise, não sendo possível clarificar a causa e o efeito entre o estresse autopercebido e demais fatores que podem desencadeá-lo. Ainda que o estresse seja uma condição comum nos idosos, seu significado é bastante variável naqueles que realizam tratamento hemodialítico, tendo em vista a multiplicidade de fatores que podem interagir entre si na causalidade dessa condição. Dessa forma, faz-se necessário, mais estudos que analisam essa temática, pois os idosos que realizam hemodiálise ficam mais sensíveis e requer um cuidado especial, para garantir um envelhecimento saudável.

\section{Referências}

1. Riella MC. Princípios de nefrologia e distúrbios hidroeletrolíticos. 5. ed. Rio de Janeiro: Guanabara Koogan, 2010.

2. Sociedade Brasileira de Nefrologia. Censo Brasileiro de Diálise 2017. [Internet]. 2018 [cited 201801 June] Available from: http://www.censo-sbn.org. br/inicio

3. Pereira E, Chemin J, Menegatti CL, Riella MC. Escolha do método dialítico - variáveis clínicas e psicossociais relacionadas ao tratamento. J. Bras. Nefrol. 2016; 38 (2): 215-224.

4. Valle $L S$, Souza VF, Ribeiro AM. Estresse e ansiedade em pacientes renais crônicos submetidos à hemodiálise. Estud. Psicol. 2013; 30(1): 131-138.

5. Guerra-Guerrero V, Sanhueza-Alvarado 0, Caceres-Espina M. Qualidade de vida de pessoas em hemodiálise crônica: relação com variáveis sociodemográficas, médico-clínicas e de laboratório. Rev. Latino-Am. Enfermagem. 2012; 20(5): [9 telas].

6. Pinto NA, Falcao EBM. Religiosidade no contexto médico: entre a receptividade e o silêncio. Rev. Bras. Educ. Med. [Internet]. 2014;38(01): 38-46.

7. Ministério da Saúde. Definição, epidemiologia e diagnóstico da DRC. Modulo 2 - UNA-SUS/ FCMA. São Luís - 2014.

8. Bonassi SM, Zângaro RA. Caracterização de Pacientes Renais Crônicos em Hemodiálise: Sinais Sintomas de Estresse. XXXIV Congresso Brasileiro de Engenharia Biomédica. CBEB. 2014

9. Arreguy-Sena C. Construção e validação de impressos: Construção e validação de impressos: sistematização do cuidado de pessoas em hemodiálise. Rev. Bras. Enferm. 2018;71(2):405-17.

10. Luft CDB. Versão Brasileira da Escala de Estresse Percebido: Tradução e validação para idosos. Rev. Saúde Pública. 2007; 41 (4): 606-15.

11. Nunes, MB, Santos EM, Leite MI, Costa AS, Guilem DB. Perfil epidemiológico de pacientes renais crônicos em programa dialítico. Rev enferm UFPE on line. 2014; 8 (1): 69-76.

12. Drackevski L, Teixeira ML. Avaliação do perfil bioquímico e parâmetros he- matológicos em pacientes submetidos a hemodiálise. Rev Saúde e Pesquisa. 2011; 4 (1): 15-22.

13. Moura L, Andrade SSCA, Malta DC, Pereira CA, Passos JEF. Prevalência de auto-relato de diagnostico médico de doença renal crônica no Brasil: pesquisa nacional de saúde, 2013. Rev Bras epidemiol. 2015; 18 (2): 181-191.

14. Melo WF, Bezerra ALD, Sousa MNA. Perfil epidemiológico de pacientes com insuficiência renal crônica: um estudo quantitativo. Rev eletrônica da Fainor. 2014; 7 (2): 142-156.

15. Melo RLP, Eulálio MC, Gouveia VV, Silva HDM. 0 efeito do estresse na qualidade de vida de idosos: o papel moderador do sentido de vida. Psicol. Reflex Crit. 2013; 26 (2):222-30.

16. Patrício ACFA, Rodrigues BFL, Ferreira MAM, Gomes RA, Brito TD, et al.Application of Mental Health Scale and Perceived Stress in Elderly Assisted by Basic Health Units. International Archives of Medicine Section: Medical Education. 2016; 9 (44): 1-7.

17. Pustiglione CCG, Mantovani MF, Nascimento MEB, Cassi CC. Social representations of illness among people with chronic kidney disease. Rev. Gaúcha Enferm. 2015; 36(2): 106-112.

18. Mattos $M$, Maruyama SAT. A experiência de uma pessoa com doença renal crônica em hemodiálise. Rev Gaúcha de Enferm. 2010; 31(3):428-34.

19. Mattos $M$, Maruyama SAT. A experiência em família de uma pessoa com diabetes mellitus e em tratamento por hemodiálise. Rev Eletrônica Enferm. 2009;11(4):971-81.

20. Kusumoto LMS, Haas VJ, Rodrigues RAP. Adultos e idosos em hemodiálise: avaliação da qualidade de vida relacionada à saúde. Acta paul. enferm. 2008; 21(spe): 152-159.

21. Milagres CS, Moraes KBD, Franceschini SCC, Sant'Ana LFR, Lima LM, Ribeiro $\mathrm{AQ}$. Prevalência e fatores associados à presença de anemia em idosos do município de Viçosa (MG), Brasil. Ciênc. saúde coletiva [Internet]. 2015 20 (12):3733-41. 